\title{
Permeability Changes of a Porous Membrane by an Adsorbed Photoresponsive Polypeptide
}

\author{
Morimasa SATo, Takatoshi KinOSHITA, Akira TAKIZAWA, \\ Yoshiharu TsujITA, and Ryuji ITO \\ Department of Materials Science \& Engineering, Nagoya Institute of Technology, \\ Gokiso-cho, Showa-ku, Nagoya 466, Japan
}

(Received February 10, 1988)

\begin{abstract}
Photoresposive polypeptides, poly(L-glutamic acid)s containing azobenzene-4sulfonic acid residues in the side chains (azo-S-X-PGA, X is the $\mathrm{mol} \%$ of the azobenzene sulfonate residue), were adsorbed onto a porous support membrane and photoinduced permeation changes of the membrane obtained were studied. The maximum amount of adsorption, $n_{\max }\left(\mathrm{g} \mathrm{g}^{-1}\right)$, of azo.S-PGA in the membrane was shown to decrease by increasing the azobenzene sulfonate groups, $\mathrm{X}$, resulting from decrease in the content of the L-glutamic acid residues which interact with the adsorption sites on the pore wall through hydrogen bonds. It was estimated that the value of $n_{\max }$ of the azo-S-14.1-PGA adsorbed membrane used for the permeation measurements was $1.40 \times 10^{-2} \mathrm{~g}$ of the polymer per $\mathrm{g}$ of the support membrane. The filtration coefficient of the azo $\cdot \mathrm{S}$ 14.1-PGA adsorbed membrane largely decreased at the $\mathrm{pH}$ where $\alpha$-helix to coil transition of the polymer occurred, indicating that permeation of the membrane was mainly determined by the conformation of the adsorbed azo-S-PGA on the pore wall. Moreover, it was observed that photoirradiation of the membrane at adequate $\mathrm{pHs}$ could induce rapid decrease in $\mathrm{Lp}$ of the membrane within a few minute. The photoresposive behavior of the membrane can be explained in terms of the photoinduced helix to coil transition of the azo-S-14.1-PGA in the membrane. The permeability of the membrane containing azo $\mathrm{S}-46$.3-PGA, which was in the random coil state at any given $\mathrm{pH}$ value in the aqueous solution, was independent on $\mathrm{pH}$ and photoirradiation.

KEY WORDS Photoresponsive Polypeptide / Azobenzene Sulfonate Side Chain / Poly(L-glutamic acid) / Adsorption / Porous Membrane / Photoinduced Conformational Change / Photoinduced Permeation Change /
\end{abstract}

Stimulus-responsive membranes have been of great interest recently, since they have characteristic functions such as transport regulation of chemical substance and/or specific signals.

In previous studies, ${ }^{1-5}$ we showed that the transport properties of polypeptide charged membranes are controlled by $\mathrm{pH}$-induced conformational change of poly(L-glutamic acid), redox-controlled conformational changes of poly(L-cystein) and photoisomerization in the side chain azobenzene moieties of azobenzenemodified poly(L-glutamic acid). Also photoinduced conformational changes of polypeptide in aqueous solution with poly(L-glutamic acid) containing azobenzene sulfonate moieties in the side chains were found. ${ }^{6}$

We report here the transport properties of a porous membrane with adsorbed poly(Lglutamic acid) containing azobenzene sulfonate moieties on the pore walls. Irradiation induced rapid changes in the volume flux through the membrane resulting from the photoinduced conformational changes of the adsorbed poly(L-glutamic acid) with azobenzene sulfonate moieties. 


\section{EXPERIMENTAL}

\section{Materials}

Poly(L-glutamic acid) (PGA, $\bar{M}_{v}=1.19 \times$ $10^{5}$ ) was obtained by saponification of poly ( $\gamma$-methyl L-Glutamate) (PMLG) as previously reported. ${ }^{7}$

Poly(L-glutamic acid) incorporated azobenzene sulfonate groups (azo $\cdot \mathrm{S}-\mathrm{X}-\mathrm{PGA}, \mathrm{X}$ is the $\mathrm{mol} \%$ of the azobenzene sulfonate moieties) was synthesized by a coupling reaction between PGA and 4-amino-1,1'-azobenzene-4'sulfonic acid sodium salt (azo $\cdot \mathrm{S})$ with dicyclohexylcarbodiimide (DCC) and $N$-hydroxybenzotriazole (HOBt) (Scheme 1). The azobenzene sulfonate content, $X$, in the polymers was determined from the absorbance at $366 \mathrm{~nm}$ of the dimethylformamide (DMF) solution of azo.S-PGAs on the basis of the molar extinction coefficient of the transform of a model compound, 4-propioamido-1,1'azobenzene- 4 '-sulfonic acid sodium salt (proazo $\cdot S)$ in DMF solution; $\lambda \max =365 \mathrm{~nm}$, $\varepsilon_{\text {max }}=2.59 \times 10^{4}$. Absorption spectra of the samples were recorded on a JASCO UVIDEC 670 spectrophotometer. The synthesis of azo.S-PGA and pro-azo.S has been described in detail previously. ${ }^{6}$

PGA with a small amount of pyrene in the side chains ( $\mathrm{PGA}^{*}$ ) was prepared to determine the amount of adsorption of PGA in the porous membrane by means of fluorescence analysis. PGA* was synthesized by the condensation reaction as follows. PGA was dissolved in DMF at $0^{\circ} \mathrm{C}$. 1-aminopyrene, $N$-hydroxybenzotriazole (HOBt) and dicyclohexylcarbodiimide (DCC) were added to the stirred DMF solution at $0^{\circ} \mathrm{C}$. After $1 \mathrm{~h}$, the mixture was further stirred for a given number of hours at $25^{\circ} \mathrm{C}$. Precipitated dicyclohexylcarbodiurea was filtered out and the bulk of the DMF was pored into ether. Then the residue obtained was washed by extraction with meth anol for 2 or 3 days. The polymer obtained, PGA*, was dried under vacuum. The pyrene content in the polymer, $2.93 \mathrm{~mol} \%$, was de-

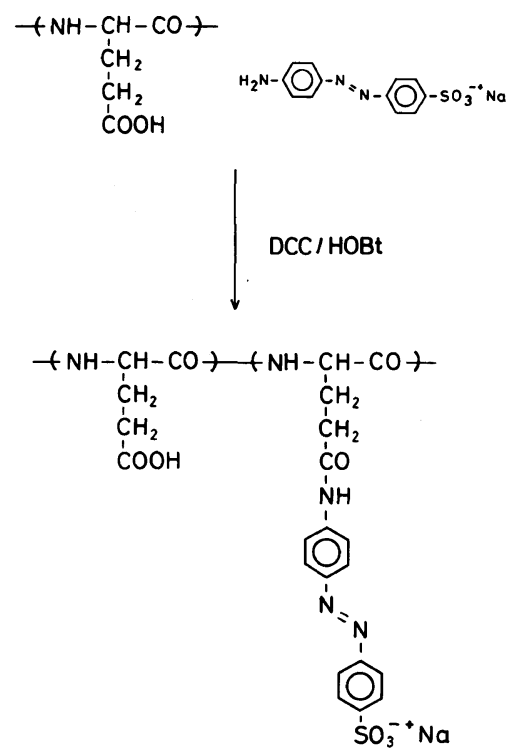

Scheme 1.

termined from the fluorescence intensity of pyrene moieties in the polymer side chains at $391 \mathrm{~nm}$ (excitation wavelength: $342 \mathrm{~nm}$ ) of the DMF solution of PGA* on the basis of the fluorescence intensity-concentration relation of pyrene monomer in DMF (the excitation wavelength of pyrene monomer was $342 \mathrm{~nm}$ and the fluorescence was observed at $386 \mathrm{~nm}$ ). Fluorescence spectroscopic mesurements were performed on the Shimadzu Co., Ltd., spectrofluorophotometer RF-540 apparatus.

\section{Preparation of Polypeptide Adsorbed Mem- branes}

Polypeptide adsorbed membranes were prepared by placing the membrane (Millipore filter, pore size of $0.1 \mu \mathrm{m}$ ) in a vessel containing aqueous solution of polypeptide $(0.42 \mathrm{~g} /$ $100 \mathrm{ml}$ ) at $\mathrm{pH} 4.5$ for PGA and at $\mathrm{pH} 3.5$ for azo $\cdot$ S-PGA without added salt at $25^{\circ} \mathrm{C}$. The adsorption equilibrium was attained within $50 \mathrm{~h}$, so that the membrane was kept in the vessel more than three days. The membrane obtained was washed with water at the equilibrium $\mathrm{pH}$ to remove non-adsorbed polypeptide in the membrane and used for the permeation 
measurements.

To elucidate the equilibrium amounts of adsorption, several pieces of the membrane were placed in an aqueous solution of polypeptide at $25^{\circ} \mathrm{C}$. After a given amount of time, one of the membranes was taken out from the solution and washed with water to remove free polypeptide from the pores of the membrane. Then the membrane was dried under vacuum and weighed. The dry membrane with adsorbed polypeptide was dissolved in DMF for spectroscopic measurements. The amounts of adsorption of azo -S-PGA and PGA (PGA*) (g of polypeptide/g of membrane) were determined from the absorbance at $366 \mathrm{~nm}$ associated with the azobenzene sulfonate moiety and fluorescence intensity at $391 \mathrm{~nm}$ associated with pyrene moiety, respectively, of the DMF solutions based on the predetermined concentration-absorbance and/or fluorescence intensity relations and the dry weight of the membrane. It should be noted that for the spectroscopic measurements the membrane material of Millipore filter co-dissolved in the DMF solutions with the polypeptides did not change in the absorbance and/or fluorescence intensity at $\lambda_{\max }$ of the polypeptides. The same procedure described above was periodically carried out until the amount of adsorption reached a constant value, the equilibrium value of the polypeptide adsorption amount.

\section{Permeation Measurements}

The measurements of volume flow across the membrane were carried out with a pyrex glass permeation cell shown in Figure 1. The polypeptide adsorbed membrane was interposed between the two parts of the cell. The volume flow across the membrane was measured under a pressure difference between the membrane of $\mathrm{ca} \cdot 10^{-2} \mathrm{~atm}$. The volume flux, $J_{v}$, was determined by observing the level of meniscus of water in the capillary tube of $0.124 \mathrm{~cm}$ radius, which was attached to the permeation side cell, using a cathetometer. The filtration coefficients, $L_{\mathrm{p}}$, were determined

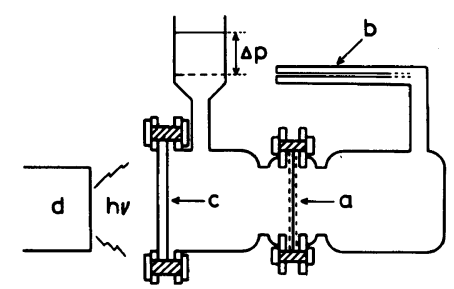

Figure 1. Experimental set-up for measuring hydrodynamic permeability of a membrane with adsorbed photoresponsive polypeptide: $a$, membrane; $b$, capillarly; c, wavelength cut-off glass filter; $d$, light source.

using $L_{\mathrm{p}}=J_{v} / \Delta P$, were $\Delta p$ is the pressure difference between the filters. Irradiation of the membrane was carried out with a $500 \mathrm{~W}$ superhigh pressure mercury lamp (Ushio UVD 500) equipped Toshiba UV-D33s filter for UV irradiation or Toshiba L-39 filter for visible light irradiation, respectively. The membrane was irradiated from the high pressure side of the cell as shown in Figure 1, since the permeability of the porous membrane was strongly dependent on the conformation of the adsorbed polymer on the pore wall near the high pressure side of the membrane rather than that near the permeated side. ${ }^{8}$

\section{Circular Dichroism (CD) Measurements}

CD spectra of the polypeptides, PGA and azo - S-PGA, were recorded on a JASCO spectropolarimeter $J 40 \mathrm{C}$ to investigate the conformation of the polypeptides in aqueous solution. The concentration of the polypeptides in aqueous solution was fixed to $c a .2 \times 10^{-4}$ $\mathrm{g} \mathrm{ml}^{-1}$. The $\mathrm{pH}$ of the solution was adjusted by the addition of $\mathrm{HCl}$ or $\mathrm{NaOH}$.

For all measurements, the membranes and solutions containing azo.S-PGA were kept in the dark for a few days to ensure that all of azobenzene moieties would be in the trans form at the begining of the measurements.

\section{RESULTS AND DISCUSSION}

\section{Polypeptide Adsorption Amounts}

The adsorption measurements for polypep- 
Table I. Maximum surface coverage, $n_{\max }$, for polypeptide-porous membrane systems

\begin{tabular}{cc}
\hline Sample & $n_{\max } / \mathrm{gg}^{-1}$ \\
\hline PGA & $3.84 \times 10^{-2}$ \\
Azo $\cdot$ S-14.1-PGA & $1.40 \times 10^{-2}$ \\
Azo $\cdot$ S-46.3-PGA & $0.75 \times 10^{-2}$ \\
\hline
\end{tabular}

tide-porous membrane systems were carried out to clarify the difference in the amount of adsorption among PGAs differing in azobenzene sulfonate content. The adsorption isotherms obtained for azo-S-14.1-PGA and azo.S-46.3-PGA are shown in Figure 2. The shapes of these isotherms were quite similar to each other, i.e., the amount of adsorption rose with increasing bulk polymer concentration and then reached a constant value. This constant value corresponding to the $\max$ imum surface coverage was attained for a bulk concentration of $c a .0 .4 \mathrm{~g} / 100 \mathrm{ml}$ almost independently of the polymer species. The values of the maximum surface coverage, $n_{\max }$, for each polypeptide are listed in Table I with that of pure PGA. It is clear that the amount of adsorption decreased with increasing azobenzene sulfonate content of the PGAs. This can be explained as follows. The adsorption of PGAs onto the porous membrane may take place through hydrogen bonds between the side chain carboxylic groups of PGAs and residual hydroxyl groups of cellulose acetate (membrane material). Therefore, the replacement of the carboxylic groups by the azobenzene sulfonate moieties does not favor proceeding adsorption through hydrogen bonds. Moreover, the pure porous membrane without polypeptide was apparently a negatively charged membrane with a fixed charge concentration of $3.40 \times 10^{-5} \mathrm{eq} / \mathrm{mol}$, estimated from membrane potential measurements. Even at low $\mathrm{pH}, 3.5$, where the adsorption of azo.SPGA was carried out, electrostatic repulsions between the polypeptide side chains and pore walls may have been present for azo-S-PGAs with sulfonate anions whose $\mathrm{p} K_{\mathrm{a}}$ was below

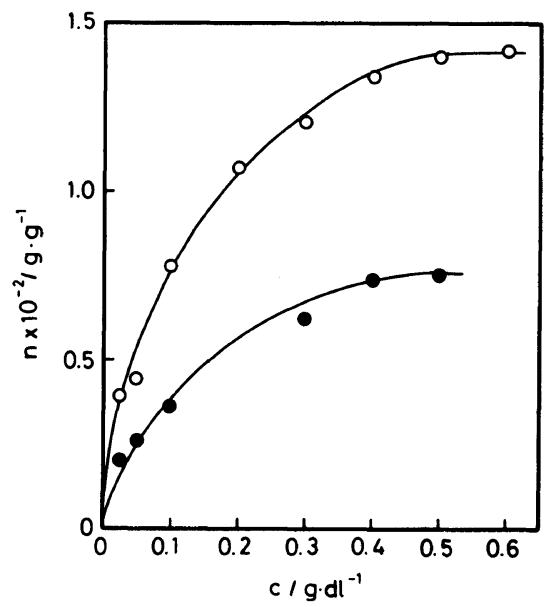

Figure 2. Adsorption isotherms of poly(L-glutamic acid) containing $14.1 \mathrm{~mol} \%(-\mathrm{O}-$ ) and $46.3 \mathrm{~mol} \%$ (-) -) of azobenzene sulfonate groups in side chain and porous support membrane systems, respectively.

1.0. Thus, the difference in the amonts of adsorption among the PGAs in Figure 2 and Table I can be ascribed to the newly incorporated photoresponsive groups into the PGA, i.e., the highly dissociable azobenzene sulfonate moieties.

pH- and Photo-Induced Conformational Changes of Azo $S$-PGA in Aqueous Solution

It is well known that the backbone structure of PGA depends on the $\mathrm{pH}$ of an aqueous solution via $\mathrm{pH}$-induced changes in th electrostatic repulsion force between dissociable Lglutamic acid side chains. The backbone structure of azo-S-PGAs with various amounts of azobenzene sulfonate moieties was examined by means of circular dichroism (CD) measurements of their aqueous solution. Figure 3 shows the (CD) spectra of the azo.S-14.1PGA in aqueous solution at various pHs. Two negative bands at $222 \mathrm{~nm}$ and $208 \mathrm{~nm}$ at $\mathrm{pH}$ 3.0 indicate that the azo.S-14.1-PGA was in the stable $\alpha$-helix structure in acid solution. The values of the molar ellipticity of both bands, however, decreased with increasing $\mathrm{pH}$ and a spectrum with a positive band at $217 \mathrm{~nm}$, associated with the random coil structure, was observed above $\mathrm{pH}$ 7.0. This indicates that 


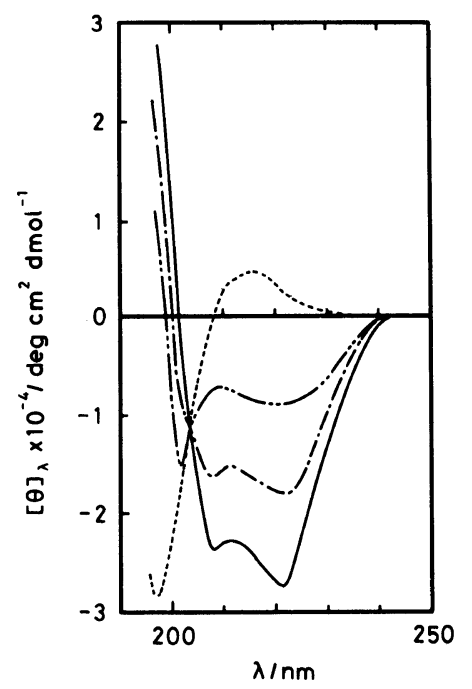

Figure 3. CD spectra of poly(L-glutamic acid) containing $14.1 \mathrm{~mol} \%$ of the azobenzene sulfonate groups in the side chains: - , at $\mathrm{pH} 3.0 ;--\ldots$, at $\mathrm{pH} 5.0 ;-\ldots$, at pH 6.0; ----, at pH 8.5.

azo $\cdot$ S-PGA containing $14.1 \mathrm{~mol} \%$ of the azobenzene sulfonate moieties shows a typical $\alpha$ helix to random coil transition in a similar manner to that of pure PGA. Figure 4 shows the $\mathrm{pH}$ dependence of molar ellipticity of the $\mathrm{CD}$ band at $222 \mathrm{~nm},[\theta]_{222}$ associated with the $\alpha$-helix content of the polypeptide, of azo.SPGAs with $14.1 \mathrm{~mol} \%$ and $46.3 \mathrm{~mol} \%$ of azobenzene sulfonate residues. It is clear that the azo.S-PGAs containing azobenzene sulfonate moieties at less than $14.1 \mathrm{~mol} \%$ changed their backbone structures from the stable righthanded $\alpha$-helix in the acid solution to the random coil by increasing the $\mathrm{pH}$ of the aqueous solution. $\mathrm{pH}$ independent small values of $[\theta]_{222}$ of azo-S-46.3-PGA showed that azo $\cdot$ S-PGA with large amounts of azobenzene sulfonate moieties were always in the random coil structure in water. This suggests that the azo sulfonate anions, whose $\mathrm{p} K_{\mathrm{a}}$ is below 1.0, in the side chains decrease the stability of the $\alpha$-helix structure via an additional electrostatic repulsion between the side chains in the azo.SPGAs even in the acid solution.

We have already shown ${ }^{6}$ that the $\alpha$-helix to (a)
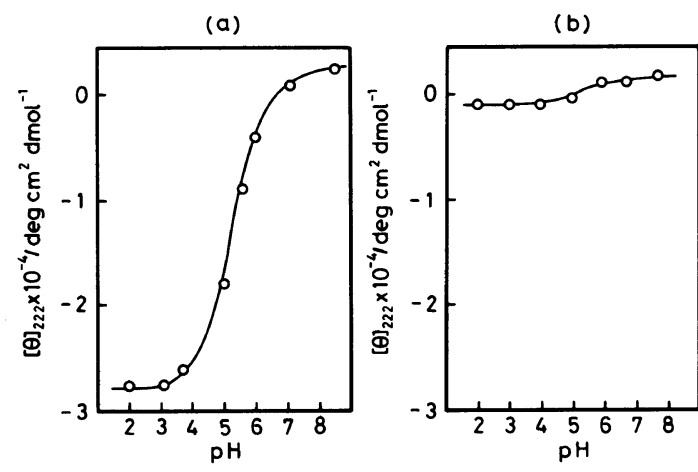

Figure 4. $\mathrm{pH}$ dependence of molar ellipticity at $222 \mathrm{~nm},[\theta]_{222}$, of poly(L-glutamic acid) containing (a): $14.1 \mathrm{~mol} \%$ and (b): $46.3 \mathrm{~mol} \%$ of azobenzene sulfonate groups in the side chains.

the coil transition of the azo-S-PGA with adequate amount of azobenzene sulfonate moieties, ca. $10 \mathrm{~mol} \%$, can be induced by UV irradiation through increase in the local charge density of the environment around the helix backbone via the photoisomerization of the side chain azobenzene chromophores from the trans to cis form. It was also shown, however, this photoinduced helix to coil transition of the azo-S-PGA was irreversible, i.e., the opposite isomerization of the azo chromophore from the cis to trans form did not affect the conformation of the azo.S-PGA in the random coil structure, since, when the backbone was in the disordered structure, relative positional changes of the azo sulfonate anions resulting from the cis to trans isomerization of the azo moieties could not induce a decrease in the local charge density of the environment around the polymer chain to effectively reproduce the original ordered structure. Figure 5(a) shows the photoinduced changes in helix content, $h(\%)$, of the azo-S-14.1-PGA at $\mathrm{pH}$ 4.0. This content was estimated from the values of $[\theta]_{222}$ obtained for the azo.S14.1-PGA assuming that $[\theta]_{222}$ of the perfect right handed $\alpha$-helix and random coil are $-3.60 \times 10^{4} \mathrm{deg} \cdot \mathrm{cm}^{2} \cdot \mathrm{dmol}^{-1}$ and $3.9 \times 10^{3}$ $\mathrm{deg} \cdot \mathrm{cm}^{2} \cdot \mathrm{d} \mathrm{mol}^{-1}$, respectively. ${ }^{9}$ The azo $\cdot \mathrm{S}$ 14.1-PGA exihibited an immediate confor- 


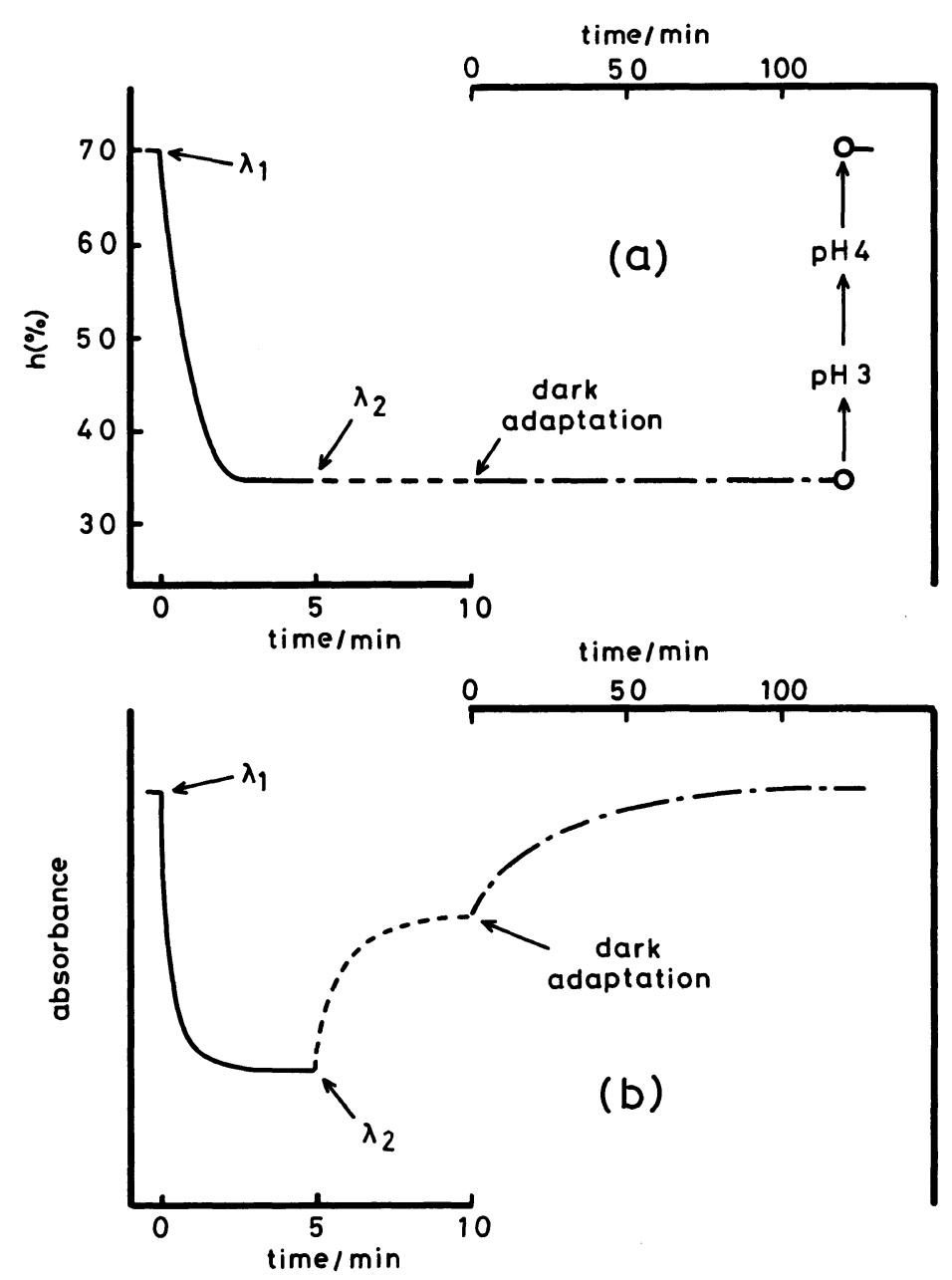

Figure 5. (a): Changes in the helix percent, $h(\%)$, of the poly(L-glutamic acid) containing $14.1 \mathrm{~mol} \%$ of azobenzene sulfonate groups in the side chains upon photoirradiation $\left(\lambda_{1}, 250 \mathrm{~nm}<\lambda_{1}<380 \mathrm{~nm}\right.$ and $\lambda_{2}$, $\lambda_{2}>390 \mathrm{~nm}$ ) and dark adaptation in aqueous solution at pH 4.0. (b): Changes in the absorbance at $354 \mathrm{~nm}$ of poly(L-glutamic acid) containing $14.1 \mathrm{~mol} \%$ of azobenzene sulfonate groups in the side chains upon photoirradiation and dark adaptation in aqueous solution at $\mathrm{pH} 4.0$.

mational response to light irradiation at $\mathrm{pH}$ 4.0 , and the helix content of the polypeptide decreased from $70 \%$ to $35 \%$ in approximately $3 \mathrm{~min}$. It was also shown that the photoinduced conformational changes of the azo.SPGA were consistent with the absorption changes of the polymer at $354 \mathrm{~nm}$, which is correlated with the trans to cis isomerization of the side chain azobenzene sulfonate moieties (Figure 5(b)). Visible light irradiation and dark adaptation, which could induce the $c$ is to trans isomerization of the azobenzene sulfonate moieties (Figure 5(b)), did not induce any structural changes of the azo.S-14.1PGA in the disordered structure (Figure 4(a)). However, the original $\alpha$-helix structure was recovered when the $\mathrm{pH}$ of the aqueous solution decreased once from 4.0 to 3.0 and then returned to 4.0 . 


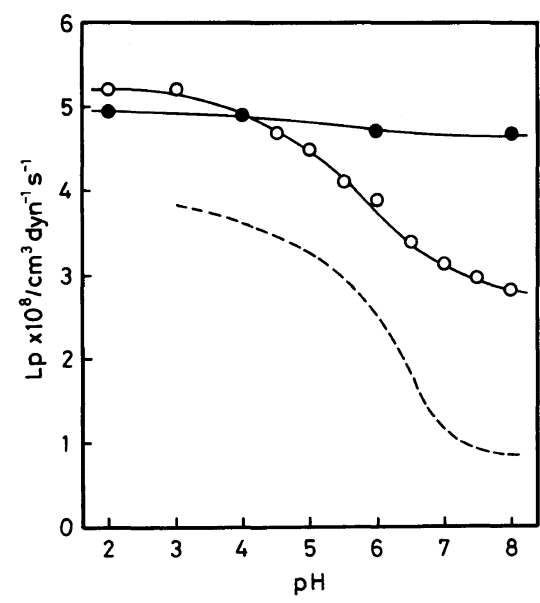

Figure 6. $\mathrm{pH}$ dependence of filtration coefficient, $L_{\mathrm{p}}$, of the polypeptide adsorbed membrane: $-\mathrm{O}-$, poly $(\mathrm{L}-$ glutamic acid) containing $14.1 \mathrm{~mol} \%$; -,$- 46.3 \mathrm{~mol} \%$ of azobenzene sulfonate groups in the side chains; ----, poly(L-glutamic acid).

\section{pH- and Photo-Induced Permeability Changes \\ Through Azo.S-PGA Adsorbed Porous Membranes}

Hydrodynamic permeability through a porous membrane with a polymer bound on the pore walls can be effectively changed by slight modification of the thickness of the adsorbed polymer layer via changes in the effective pore radius for water permeation. Pefferkorn et $a l .{ }^{10}$ have already shown that the hydrodynamic permeability through the membrane with adsorbed pure PGA steeply decreases at the $\mathrm{pH}$ where the helix to coil transition of the polymer occurs at the interface.

Figure 6 shows the $\mathrm{pH}$ dependence of the filtration coefficients, $L_{\mathrm{p}}$, of the membrane with the pure PGA, azo.S- 14.1-PGA and azo.S-46.3-PGA, respectively, bound on the pore walls. The magnitude of $L_{\mathrm{p}}$ of the membrane with azo.S-PGAs was shown to be the following order in the $\mathrm{pH}$ above 7.0, where each polypeptide was in the same structure, random coil conformation: azo-S-46.3PGA $>$ azo $\cdot$ S-14.1-PGA $>$ pure PGA, indicating that the effective pore radius of the membrane increases with increasing azo sulfonate content of the azo-S-PGAs adsorbed in the membrane. This order can be explained by the difference in the amount of adsorption of azo.S-PGAs in the membrane, i.e., the equilibrium amount of adsorption of the azo-S-PGA was decreased with increasing azo sulfonate content of the polymer (Figure 2 and Table I). Moreover, it is found that the hydrodynamic permeability of the membrane with the azo $\cdot \mathrm{S}$ 46.3-PGA was almost independent of the $\mathrm{pH}$ of the aqueous solution. This is consistent with the fact that the backbone structure of the azo $\cdot$ S-46.3-PGA dose not depend on the $\mathrm{pH}$ value in the aqueous solution (Figure 4). The $L_{\mathrm{p}}$ of the membranes with pure PGA and azo.S-14.1-PGA was strongly dependent on the $\mathrm{pH}$; i.e., the permeability slightly decreased with increasing $\mathrm{pH}$ in acid solution and steeply decreased at the conformational transition $\mathrm{pH}$ and then slightly decreased again from neutral to weak alkaline $\mathrm{pH}$. The inflexion point on the $\mathrm{pH}$ dependence of $L_{\mathrm{p}}$ of the membrane with azo-S-14.1-PGA is at a $\mathrm{pH}$ lower than that of a membrane with pure PGA, reflecting the fact that the $\mathrm{pH}$ at which the conformational transition of azo-S-14.1-PGA occures shifts to lower $\mathrm{pH}$ value. Thus, the $\mathrm{pH}$ response of the permeability of the membranes is closely correlated with the conformational behavior of the azo.S-PGAs in aqueous solution. Figure 7 shows relative variations with $\mathrm{pH}$ of the $L_{\mathrm{p}},\left(L_{\mathrm{p}}-L_{\mathrm{p}}(\mathrm{pH})\right) /\left(L_{\mathrm{p}}(\mathrm{pH})\right.$ $\left.-L_{\mathrm{p}}(\mathrm{pH} 8)\right)$, of the membrane with azo $\cdot \mathrm{S}$ 14.1-PGA and helix content, $(h-h(\mathrm{pH}$ $8) /(h(\mathrm{pH} 2)-h(\mathrm{pH} 8))$, of azo $\cdot \mathrm{S}-14.1-\mathrm{PGA}$ in aqueous solution, respectively. The changes in $L_{\mathrm{p}}$ occurred over a $\mathrm{pH}$ range wider than the conformatinal changes in solution; however, the variation of both parameters was nearly the same, indicating that $\mathrm{pH}$ response of permeability can be attributed to the interfacial conformational transition of the azo.S-14.1PGA on the pore walls.

In the next step of controlling the per meability of the membrane, a conformational transition of the azo-S-PGA on the pore walls 


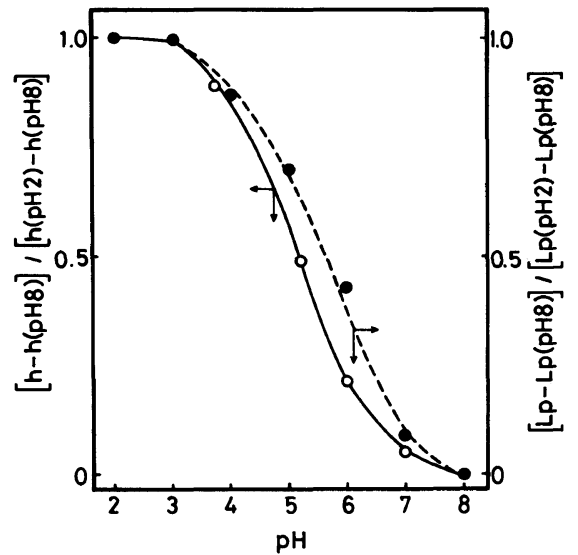

Figure 7. $\mathrm{pH}$ dependence of $\left[L_{\mathrm{p}}-L_{\mathrm{p}}(\mathrm{pH})\right] /\left[L_{\mathrm{p}}(\mathrm{pH}\right.$ 2) $-L_{\mathrm{p}}(\mathrm{pH} 8)$, - - - of the membrane with adsorbed poly(L-glutamic acid) containing $14.1 \mathrm{~mol} \%$ of azobenzene sulfonate groups in the side chains and $[h-h(\mathrm{pH}$ $8)] /[h(\mathrm{pH} 2)-h(\mathrm{pH})],-\mathrm{O}-$, of poly(L-glutamic acid) containing $14.1 \mathrm{~mol} \%$ of azobenzene sulfonate groups in the side chains in aqueous solution.

was induced by light irradiation. The dependence of the filtration coefficient, $L_{\mathrm{p}}$, on UV light irradiation was observed at $\mathrm{pH} 4.0$, using the membrane with azo.S-14.1-PGA on the pore walls (Figure 8 ). $L_{\mathrm{p}}$ decreased by irradiation within $3 \mathrm{~min}$. Dark adaptation did not induce any change in $L_{\mathrm{p}}$ of the membrane, reflecting the irreversibility of the photoinduced conformational transition of the azo $\cdot \mathrm{S}$ 14.1-PGA. However, the $L_{\mathrm{p}}$ returned to the initial value adjusting the aqueous medium once to $\mathrm{pH} 3.0$ and then to $\mathrm{pH} \mathrm{4.0.} \mathrm{It} \mathrm{is} \mathrm{clear}$ that the changes in $L_{\mathrm{p}}$ in Figure 8 are entirely consistent with the photo- and $\mathrm{pH}$-induced conformational transition of the azo.S-14.1PGA in aqueous solution (Figure 5(a)). In addition, Figure 9 shows photoinduced variation of the parameters, $\Delta L_{\mathrm{p}}$ and $\Delta h(\%)$, defined, by

$\Delta L_{\mathrm{p}}=L_{\mathrm{p}}$ on UV irradiation $-L_{\mathrm{p}}$ in the dark $\Delta h(\%)=h(\%)$ on UV irradiation $-h(\%)$ in the dark

where $h(\%)$ is the helix content of the polymer in aqueous solution. It is clear that UV light

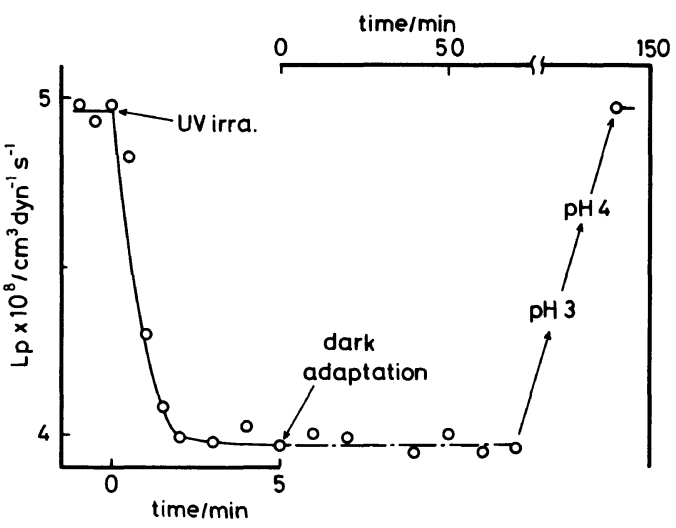

Figure 8. Changes in $L_{\mathrm{p}}$ of the membrane with adsorbed poly(L-glutamic acid) containing $14.1 \mathrm{~mol} \%$ of azobenzene sulfonate groups in the side chains on ultraviolet light irradiation $(250 \mathrm{~nm}<\lambda<380 \mathrm{~nm})$ and the dark adaptation at $\mathrm{pH} 4.0$.
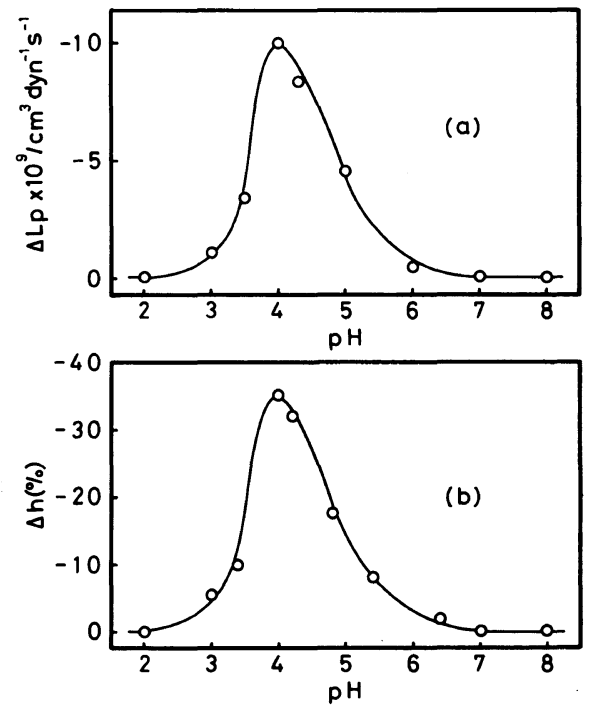

Figure 9. (a): $\mathrm{pH}$ dependence of $\Delta L_{\mathrm{p}}\left(=L_{\mathrm{p}}\right.$ on UV irradiation $-L_{\mathrm{p}}$ in the dark) of the adsorbed membrane with poly(L-glutamic acid) containing $14.1 \mathrm{~mol} \%$ of azobenzene sulfonate groups in the side chains. (b): $\mathrm{pH}$ dependence of $\Delta h(\%)(=h(\%)$ on UV irradiation $-h$ $(\%)$ in the dark) of poly(L-glutamic acid) containing 14.1 mol $\%$ of azobenzene sulfonate groups in the side chains in aqueous solution. Irradiation was carried out for $10 \mathrm{~min}$.

irradiation dose not induce any change in $L_{\mathrm{p}}$ at the lower pHs $(<3.0)$ and higher $\mathrm{pHs}(>7.0)$ (Figure 9(a)), where conformation of the 
azo.S-14.1-PGA was also insensitive to light (Figure 9(b)). As a result, the photoresponsive transport can be observed in the relatively narrow $\mathrm{pH}$ range where photoinduced conformational transition of the azo-S-14.1-PGA occurs. A similar result was also obtained with the membrane containing azo-S-9.3-PGA on the pore walls. Therefore, the rapid changes in the hydrodynamic permeability of the membrane with azo-S-PGA containing $c a .10$ $\mathrm{mol} \%$ of azo sulfonate moieties can be induced by UV light irradiation at adequate pHs due to interfacial conformational transition of the azo-S-14.1-PGA based on the trans to cis isomerization of the side chain azo sulfonate moieties by irradiation.

Acknowledgement. The authors gratefully acknowledge the support of the Japanese Ministry of Education through a Grant-in-Aid for Developmental Science Research.

\section{REFERENCES}

1. T. Kinoshita, A. Takizawa, and Y. Tsujita, Nippon Kagaku Kaishi, 868 (1983).

2. A. Takizawa, T. Kinoshita, A. Ohtani, and Y. Tsujita, J. Polym. Sci., Polym. Chem. Ed., 24, 665 (1986).

3. T. Kinoshita, M. Sato, A. Takizawa, and Y. Tsujita, J. Chem. Soc., Chem. Commun., 929 (1984).

4. A. Takizawa, M. Sato, T. Kinoshita, and Y. Tsujita, Chem. Lett., 1963 (1984).

5. T. Kinoshita, M. Sato, A. Takizawa, and Y. Tsujita, Macromolecules, 19, 51 (1986).

6. M. Sato, T. Kinoshita, A. Takizawa, and Y. Tsujita, Macromolecules, 21, 1612 (1988).

7. T. Kinoshita, T. Yamashita, T. Iwata, A. Takizawa, and Y. Tsujita, J. Macromol. Sci., Phys. Ed., B22, 1 (1983).

8. A. Takizawa, T. Kinoshita, Y. Tsujita, and S. Ito, Membrane, 9, 349 (1984).

9. N. Greenfield and G. D. Fasman, Biochemistry, 8, 4108 (1969).

10. E. Pefferkorn, A. Schmitt, and R. Varoqui, Biopolymers, 21, 1451 (1982). 